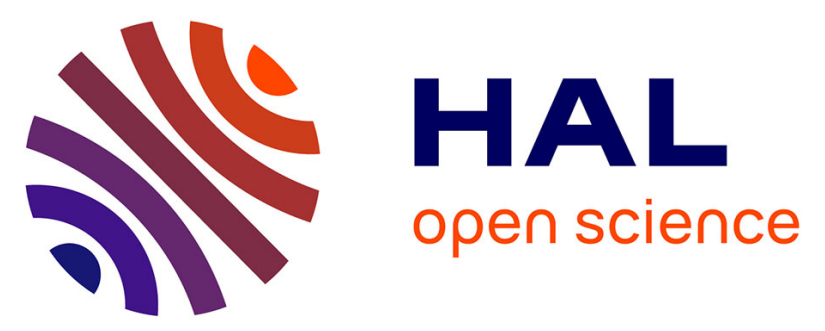

\title{
TiO2 microsphere-based metamaterials exhibiting effective magnetic response in the terahertz regime
}

Riad Yahiaoui, Mario Maglione, Christelle Kalde, Filip Kaldec, Petr Kužel, U-Chan Chung, Catherine Elissalde

\section{- To cite this version:}

Riad Yahiaoui, Mario Maglione, Christelle Kalde, Filip Kaldec, Petr Kužel, et al.. TiO2 microspherebased metamaterials exhibiting effective magnetic response in the terahertz regime. Applied physics. A, Materials science \& processing, 2012, 109 (4), pp.891-894. 10.1007/S00339-012-7356-X . hal00752457

\section{HAL Id: hal-00752457 \\ https://hal.science/hal-00752457}

Submitted on 6 Dec 2012

HAL is a multi-disciplinary open access archive for the deposit and dissemination of scientific research documents, whether they are published or not. The documents may come from teaching and research institutions in France or abroad, or from public or private research centers.
L'archive ouverte pluridisciplinaire HAL, est destinée au dépôt et à la diffusion de documents scientifiques de niveau recherche, publiés ou non, émanant des établissements d'enseignement et de recherche français ou étrangers, des laboratoires publics ou privés. 


\title{
$\mathrm{TiO}_{2}$ microsphere-based metamaterials exhibiting effective magnetic response in the terahertz regime
}

\author{
R. Yahiaoui • H. Němec $\cdot$ C. Kadlec $\cdot$ F. Kadlec $\cdot$ \\ P. Kužel • U.-C. Chung • C. Elissalde • M. Maglione • \\ P. Mounaix
}

Received: 15 February 2012 / Accepted: 10 October 2012 / Published online: 1 November 2012

(C) Springer-Verlag Berlin Heidelberg 2012

\begin{abstract}
Thin layers of all-dielectric metamaterials based on $\mathrm{TiO}_{2}$ spherical particle resonators are investigated. A new method based on spray drying of dissolved nanoparticles is used in the fabrication process. Spectral footprints of electric and magnetic dipoles are reported numerically and through experimental tests. It is a promising step for the construction of novel three-dimensional isotropic metamaterials exhibiting desired electromagnetic properties for terahertz applications.
\end{abstract}

\section{Introduction}

Since their advent, metamaterials have been explored for their potential use in sub-diffraction-limited imaging [1], medical imaging [2], sensing applications [3], cloaking [4], and so on. We focus in this paper on the study of alldielectric (AD) metamaterials based on layers of titanium dioxide $\left(\mathrm{TiO}_{2}\right)$ microspheres. Their effective electric and magnetic responses are related to Mie resonances in each

\footnotetext{
R. Yahiaoui $(\varangle) \cdot$ P. Mounaix

LOMA, Université Bordeaux 1, CNRS, UMR 5798, 33400

Talence, France

e-mail: r.yahiaoui@ loma.u-bordeaux1.fr

H. Němec · C. Kadlec · F. Kadlec · P. Kužel

Institute of Physics, Academy of Sciences of the Czech Republic,

Na Slovance 2, 18221 Prague 8, Czech Republic

U.-C. Chung $\cdot$ C. Elissalde $\cdot$ M. Maglione

ICMCB, Université Bordeaux, CNRS - UPR 9048, 87 Avenue du

Docteur Albert Schweitzer, 33608 Pessac cedex, France

U.-C. Chung

CRPP, CNRS - UPR 8641, 115 Avenue du Docteur Albert

Schweitzer, 33600 Pessac cedex, France
}

single particle. For high-enough dielectric permittivity of the particles, it is then possible to achieve e.g. negative effective magnetic permeability [5]. Although AD metamaterials have raised broad interests [6], only a few of them have been experimentally shown to present a magnetic resonance in the $\mathrm{THz}$ spectral range. For example, laser micromachining was employed to fabricate a tunable $\mathrm{THz}$ metamaterial made of $\mathrm{SrTiO}_{3}$ rods [7]. The majority of other works focused on the microwave region. For instance, Zhao et al. demonstrated experimentally a negative magnetic permeability at microwave frequencies in a three-dimensional dielectric composite metamaterial based on high dielectric permittivity cubic particles of $\mathrm{Ba}_{0.5} \mathrm{Sr}_{0.5} \mathrm{TiO}_{3}$ (BST) coated with Teflon (PTFE) [8]. Vendik and Gashinova [9] in their work investigated theoretically and numerically a periodic composite medium consisting of two lattices of dielectric spherical particles with different diameters or dielectric constants at microwave frequencies. The authors showed that an effective isotropic double negative medium (DNG) can be realized in the frequency region where the resonance of the TM mode in one kind of particles and of the TE mode in the other kind of particles are achieved simultaneously. Moreover, Peng et al. [10] observed experimentally a left-handed behavior at gigahertz frequencies in an array of standard dielectric resonators. Lepetit et al. [11] have measured a negative refractive index in an all-dielectric metamaterial in the microwave frequency range. Shibuya et al. [12] predicted theoretically a left-handed behavior in a lattice combining two sets of $\mathrm{TiO}_{2}$ cubes with two different sizes.

\section{Our investigated metamaterials}

In this work, inexpensive methods were used to fabricate monodisperse micron-sized spheres. First, we mixed com- 


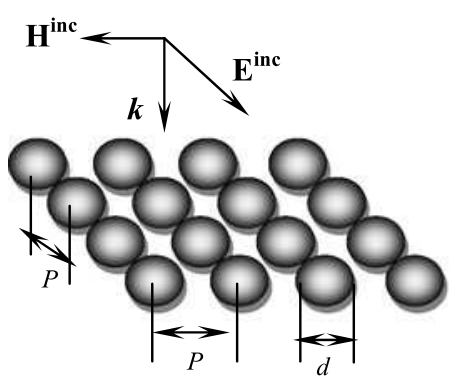

$\mathbf{a}$

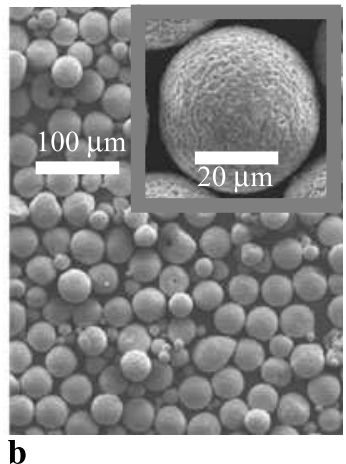

Fig. 1 (a) Schematic view of the simulated microsphere-based metamaterial, with the relevant geometrical dimensions: diameter $d=45 \mu \mathrm{m}$, period $P=103 \mu \mathrm{m}$, and the appropriate electric and magnetic field polarizations. (b) Scanning electron microscope images of our $\mathrm{TiO}_{2}$ microspheres before the sorting procedure

mercial $\mathrm{TiO}_{2}$ nanoparticles with ethanol to obtain a liquid suspension, which was suddenly dried upon spraying through a flame. This resulted in assembling $\mathrm{TiO}_{2}$ nanoparticles into fragile mostly spherical dense clusters. These microspheres were then sintered in a tube furnace at $1200{ }^{\circ} \mathrm{C}$ for $2 \mathrm{~h}$, in order to solidify them and to minimize their porosity. A scanning-electron-microscope image of these particles is shown in Fig. 1b.

The microspheres were finally sieved and sorted along their diameters $d$. We investigated powders with the following diameters: (i) $\mathrm{MS}_{<38}, d<38 \mu \mathrm{m}$, (ii) $\mathrm{MS}_{38-40}$, $38 \mu \mathrm{m}<d<40 \mu \mathrm{m}$, and (iii) $\mathrm{MS}_{40-50}, 40 \mu \mathrm{m}<d<50 \mu \mathrm{m}$.

\section{Result of numerical and experimental characterization}

We measured the complex transmission and reflection of the samples by terahertz time domain spectroscopy (THzTDS) to retrieve the effective electromagnetic response of the metamaterial structures. A thin layer of $\mathrm{TiO}_{2}$ microparticles has been spread randomly between two thick blocks of sapphire separated by a $70 \mu \mathrm{m}$ thick Teflon o-ring. This fixes and controls the thickness of the film, which is then essentially composed of a single layer of the microparticles.

$\mathrm{THz}$ pulses passing through the structure directly carry information about the complex transmittance of the powder, while $\mathrm{THz}$ pulses coming from internal reflections in the blocks and from the partial reflection on the sapphire/powder interface carry information also about the complex reflectance of the powder (Fig. 2a). These pulses are resolved as a sequence of echoes in the time-domain signal transmitted through the entire structure [13] (see Fig. 2b). The sapphire block B is about two times thicker than the block A, which ensures that the first internal reflection in the block A does not mix with the internal reflections
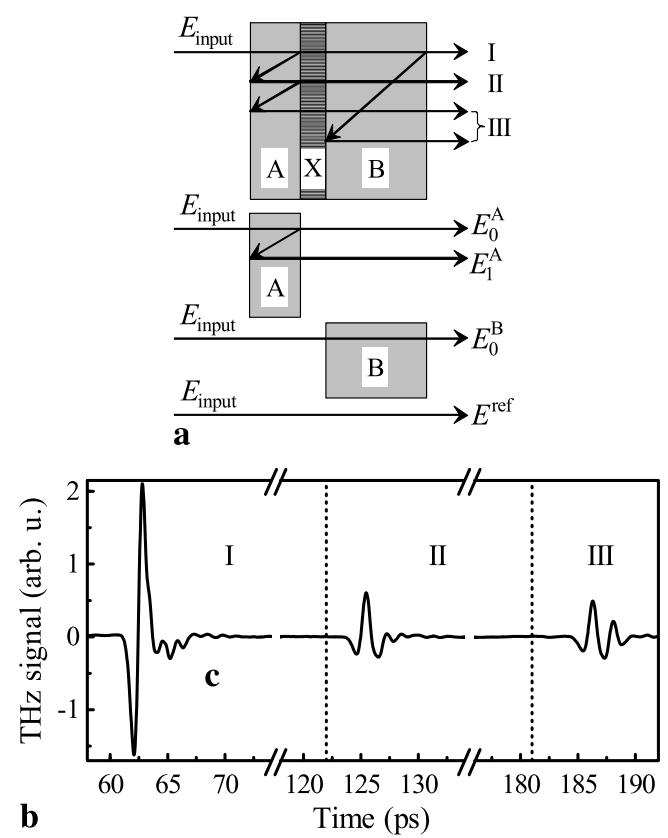

Fig. 2 (a) Scheme of the pulse propagation through the structure AXB (sapphire-powder-sapphire) and within the three associated reference measurements. The reflected beams are shifted vertically for graphical clarity. (b) Example of the time-domain signal transmitted through the structure AXB. Dotted lines delimit the intervals containing: I-main pulse $\left(E_{0-A X B}\right)$; II-1st echo in A $\left(E_{1-A X B}\right)$; III-superposition of 1st echo in $\mathrm{B}$ and 2 nd echo in $\mathrm{A}$

from the block B. The measurement has to be supplemented by three reference measurements: (i) waveform transmitted through the block A (including the first echo), (ii) waveform transmitted through the block B, and (iii) waveform transmitted through an empty space. The complex transmittance and reflectance spectra of the metamaterial are then calculated from

$t=\frac{E_{0}^{\mathrm{AXB}} E^{\mathrm{ref}}}{E_{0}^{\mathrm{A}} E_{0}^{\mathrm{B}}} \cdot \frac{4 z_{\mathrm{B}}}{\left(1+z_{\mathrm{A}}\right)\left(1+z_{\mathrm{B}}\right)}$,

$r=\frac{E_{1}^{\mathrm{AXB}} E_{0}^{\mathrm{A}}}{E_{1}^{\mathrm{A}} E_{0}^{\mathrm{AXB}}} \cdot \frac{1-z_{\mathrm{A}}}{1+z_{\mathrm{A}}}$,

where $z_{\mathrm{A}}$ and $z_{\mathrm{B}}$ are the relative wave impedances of the blocks $\mathrm{A}$ and $\mathrm{B}$, respectively, and $E$ denotes the Fourier transformations (spectra) of the time-domain signals defined in Fig. 2a. The use of the same blocks in the reference measurements ensures that the transmittance and reflectance phase is not corrupted by a possible uncertainty in the determination of the thickness of these blocks. We used thick sapphire blocks ( 3 and $6 \mathrm{~mm}$ ). The internally reflected pulses (echoes) are thus separated by more than 60 ps (see dotted lines in Fig. 2b), which enables a good spectral resolution $(1 /(60 \mathrm{ps}) \approx 0.03 \mathrm{THz})$. The transmission and reflection spectra are then extracted numerically as shown in Fig. 3. In the related numerical simulations, we considered a periodic array of monodisperse spheres $(d=45 \mu \mathrm{m}$, filling factor $=$ 
Fig. 3 Spectra of the sample $\mathrm{MS}_{40-50}$ : simulated (solid lines) and measured (hollow circles) magnitude of the reflection (a), phase of the reflection (b), magnitude of the transmission (c), phase of the transmission (d)
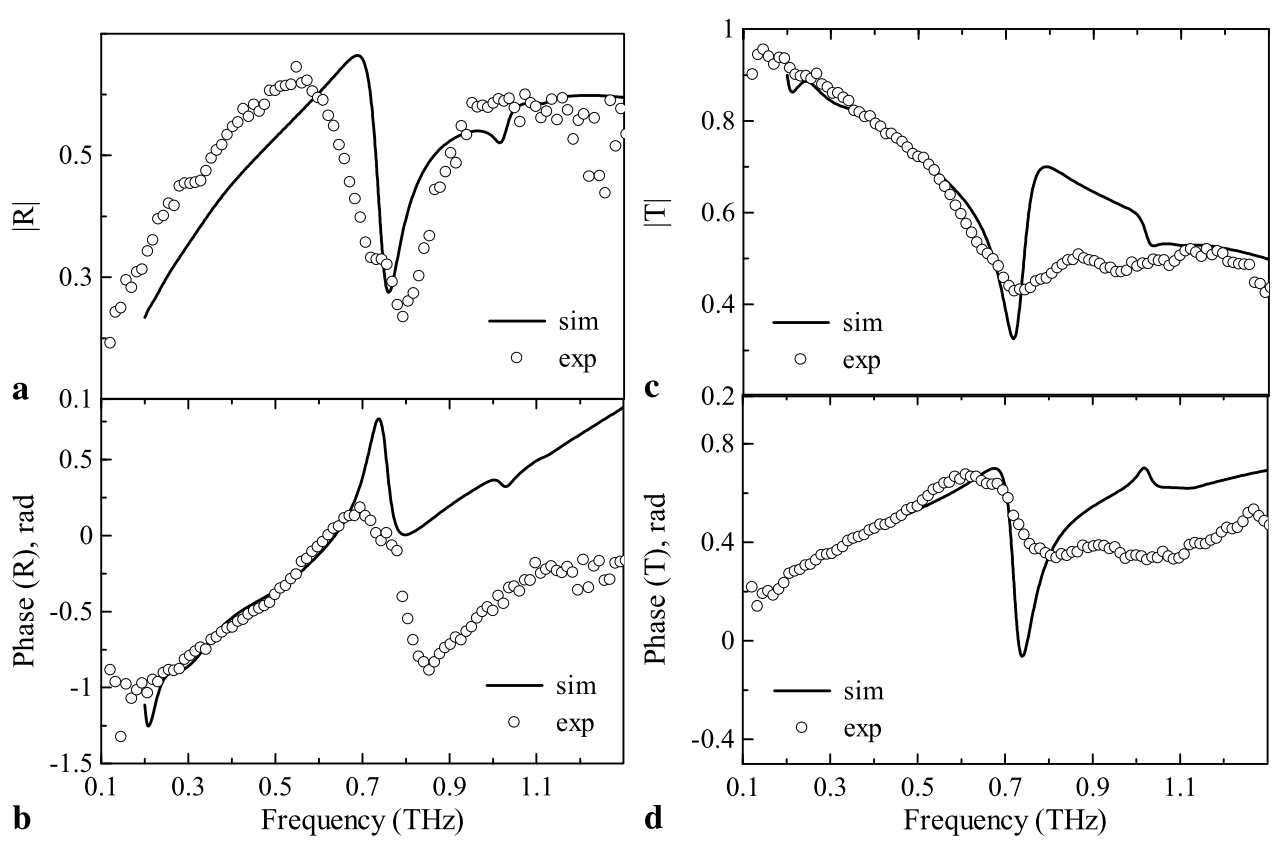

$10 \%$ ), as illustrated in Fig. 1a. We found that $\mathrm{TiO}_{2}$ microparticles have a relative dielectric permittivity of about 92 and a moderate loss level of about $5 \%(\tan \delta \sim 0.05)$ at terahertz frequencies. The overall agreement between simulation and experiment is rather good, keeping in mind that a regular structure is simulated while the measured sample is necessarily irregular and the microspheres may touch each other. We notice minor differences in the simulated phases compared to the measured ones (cf. Fig. 3b and d) above the first Mie mode around $0.75 \mathrm{THz}$, which is probably due to the polydispersity of the microspheres.

\section{Retrieval of the effective electromagnetic parameters}

Using the retrieval method based on the inversion of Fresnel equations [14], we determined the effective permittivity and permeability. The result of the extraction procedure is given in Fig. 4. There is a good agreement between the simulated and the measured effective magnetic responses (Fig. 4b). We believe that magnetic resonances are excited individually within each particle forming the metamaterial and they are only weakly influenced by the mutual coupling between particles [7, 16, 17]. Both measured and calculated effective dielectric permittivities are rather low (Fig. 4a), which is due to the low filling factor (about $10 \%$ ) and low percolation degree which is controlled by the actual structure of the film [15].

Furthermore, the calculated permittivity at low frequencies is significantly lower than the measured one and the resonance observed here is not as strong as the measured one. This difference between simulation and experiment can be partly explained by the complex topology of our samples (note that a simple square lattice of microspheres was considered in the simulations represented in Fig. 1a). In order to attempt reproducing the resonant behavior of the electric response, we performed further numerical calculations taking into account pairs of microspheres in contact (dimers) arranged in a rectangular lattice, as illustrated in Fig. 4c. The resonant behavior, similar to the experimental one shown in Fig. $4 d$, is then observed in the permittivity spectra for a probing electric field parallel to the dimer axis.

On the one hand, polydispersity of the spheres in samples broadens the resonance and prevents $\mu$ from reaching negative values. On the other hand, numerical simulations show that using a much larger filling fraction (e.g. $50 \%$ ) provides a metamaterial with $\mu<0$ in spite of the inhomogeneous broadening. This implies that the microparticles fabricated by our method can induce a negative-permeability metamaterial for large filling fractions. Unfortunately, the high absorption for filling fractions exceeding $\sim 15 \%$ precluded an accurate experimental determination of the permeability in such structures.

\section{Conclusions}

In conclusion, $\mathrm{TiO}_{2}$ microparticle-based metamaterials were fabricated using an inexpensive spray-drying method. $\mathrm{Nu}$ merical calculations and experimental characterizations have been conducted, demonstrating effective electric and magnetic response at terahertz frequencies, due to Mie resonances. It is a promising step for implementing improved electromagnetic functionalities, involving cheap and easily made metamaterials. 

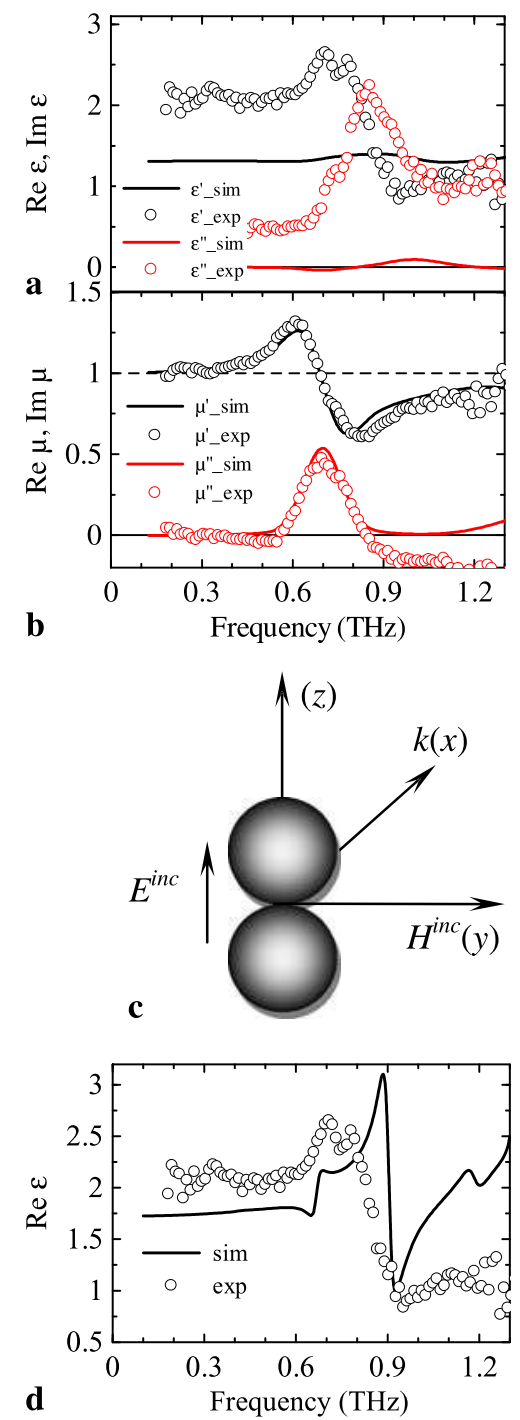

Fig. 4 Dielectric permittivity (a) and magnetic permeability (b) of the sample $\mathrm{MS}_{40-50}$. Circles: measured data, lines: simulations incorporating inhomogeneous broadening. (c) Configuration of the dimer, with the probing electric field parallel to its axis. (d) Real part of the dielectric permittivity of the dimer (sphere diameter $50 \mu \mathrm{m}$, filling factor $15 \%$ ) compared to the experimental permittivity from (a)
Acknowledgements The work at the University of Bordeaux 1 was supported by the project 'GIS-AMA-SAMM'. Financial support by the Czech Science Foundation (Project No. P204/12/0232) is also acknowledged.

\section{References}

1. N. Fang, H. Lee, C. Sun, X. Zhang, Science 308, 534 (2005)

2. M.J. Freire, R. Marques, L. Jelinek, Appl. Phys. Lett. 93, 231108 (2008)

3. K. Kneipp, H. Kneipp, I. Itzkan, R.R. Dasari, M.S. Feld, J. Phys. Condens. Matter 14, 597 (2002)

4. D. Schurig, J.J. Mock, B.J. Justice, S.A. Cummer, J.B. Pendry, A.F. Starr, D.R. Smith, Science 314, 977 (2006)

5. S. O’Brien, J.B. Pendry, J. Phys. Condens. Matter 14, 4035 (2002)

6. Q. Zhao, J. Zhou, F. Zhang, D. Lippens, Mater. Today 12, 60 (2009)

7. H. Němec, P. Kužel, F. Kadlec, C. Kadlec, R. Yahiaoui, P. Mounaix, Phys. Rev. B 79, 241108 (2009)

8. Q. Zhao, L. Kang, B. Du, H. Zhao, Q. Xie, X. Huang, B. Li, J. Zhou, L. Li, Phys. Rev. Lett. 101, 027402 (2008)

9. O.G. Vendik, M.S. Gashinova, Artificial double negative (DNG) media composed by two different dielectric sphere lattices embedded in a dielectric matrix, in Proc. 34th Eur. Microwave Conf., Amsterdam (2004), pp. 1209-1212

10. L. Peng, L. Ran, H. Chen, H. Zhang, J.A. Kong, M.T. Grzegorczyk, Phys. Rev. Lett. 98, 157403 (2007)

11. T. Lepetit, E. Akmonsoy, J.-P. Ganne, Appl. Phys. Lett. 95, 121101 (2009)

12. K. Shibuya, K. Takano, N. Matsumoto, K. Izumi, H. Miyazaki, Y. Jimba, M. Hangyo, Terahertz metamaterials composed of $\mathrm{TiO}_{2}$ cube arrays, in Proc. Metamaterials, Pamplona (2008), pp. 777779

13. L. Duvillaret, F. Garet, J.-L. Coutaz, Appl. Opt. 38, 409 (1999)

14. D.R. Smith, S. Schultz, P. Markoš, C.M. Soukoulis, Phys. Rev. B 65, 195104 (2002)

15. H. Němec, Z. Mics, M. Kempa, P. Kužel, O. Hayden, Y. Liu, T. Bein, D. Fattakhova-Rohlfing, J. Phys. Chem. C 115, 6968 (2011)

16. H. Němec, C. Kadlec, F. Kadlec, P. Kužel, R. Yahiaoui, U.-C. Chung, C. Elissalde, M. Maglione, P. Mounaix, Appl. Phys. Lett. 100, 061117 (2012)

17. R. Yahiaoui, H. Němec, P. Kužel, F. Kadlec, C. Kadlec, P. Mounaix, Opt. Lett. 34, 3541 (2009) 